Paper

\title{
Remarks on computable a priori error estimates for finite element solutions of elliptic problems
}

\author{
Akitoshi Takayasu ${ }^{1}$, Xuefeng Liu ${ }^{2 a)}$, and Shin'ichi Oishi ${ }^{1,3}$ \\ ${ }^{1}$ Department of Applied Mathematics, Faculty of Science and Engineering, \\ Waseda University \\ 3-4-1 Okubo, Shinjuku, Tokyo 169-8555, Japan \\ ${ }^{2}$ Research Institute for Science and Engineering, Waseda University \\ 3-4-1 Okubo, Shinjuku, Tokyo 169-8555, Japan \\ ${ }^{3}$ CREST/JST \\ a)xfliu.math@gmail.com
}

Received May 20, 2013; Revised August 21, 2013; Published January 1, 2014

\begin{abstract}
For Poisson's equation over a polygonal domain of general shape, the solution of which may have a singularity around re-entrant corners, we provide an explicit a priori error estimate for the approximate solution obtained by finite element methods of high degree. The method used herein is a direct extension of the one developed in preceding paper of the second and third listed authors, which provided a new approach to deal with the singularity by using linear finite elements. In the present paper, we also give a detailed discussion of the dependency of the convergence order on solution singularities, mesh sizes and degrees of the finite element method used.
\end{abstract}

Key Words: Finite element method, a priori error estimate, verified numerical computation, Poisson's equation, hypercircle equation

\section{Introduction}

The goal of this paper is to apply a finite element method (FEM) of high degree to obtain a significantly improved a priori estimate for the solution to the boundary value problem of Poisson's equation over a polygonal domain $\Omega$ :

$$
-\Delta u=f \text { in } \Omega ; \quad u=0 \text { on } \partial \Omega .
$$

For the above boundary value problem, FEMs are efficient tools for calculating an approximate solution. Estimating the approximation error is a fundamental problem in both industrial applications and theoretical analysis. In particular, for non-linear equations, e.g., $f$ is a non-linear function of $u$, explicit error estimates are highly desirable for verification of the existence of solutions. Furthermore, when the domain under consideration has a re-entrant corner, the solution has a singularity there, making explicit a priori error estimates somewhat difficult. 
In [2], N. Yamamoto and M.T. Nakao provided an explicit a priori error estimate for Poisson's equation over an L-shaped domain. However, the techniques used in the error estimates therein cannot easily be applied to domains of more general shape. More recently, X. Liu and S. Oishi [1] successfully adopted a mixed FEM and a conforming FEM to compute an a priori error estimate for a polygonal domain. The main idea of [1] is to utilize the hypercircle equation originating from [10] to handle the singularity, and their method can be applied to solve the problem over more general domains without additional effort.

In this paper, we extend the method of X. Liu and S. Oishi to consider high degree FEMs and give an improved a priori error estimate. Due to the singularity of the solution in the case of a non-convex domain, a finite element method of higher degree cannot give a higher convergence order. However, if we use a FEM in which we vary both the mesh size $(h)$ and shape function degree $(p)$, which is called by HP-FEM [3], an improved error estimate becomes possible. Our sample numerical computations demonstrate that implementing HP-FEMs can give very fast convergence versus the degrees of freedom of the FEM space.

The outline of this paper is as follows: in Section 2, we give the preliminary definitions and results on function spaces and problems needed in the sequel; in Section 3, we prove the main theorem for a priori error estimates for high degree FEMs; in Section 4, we provide computations over several nonconvex domains to illustrate our results; in the final section, we give an application of our proposed estimate to the verification of the existence of solution for a semilinear elliptic equation.

\section{Preliminaries}

Given polygonal domain $\Omega \subset \mathbf{R}^{2}$, the Sobolev spaces $H^{r}(r \in \mathbf{N})$ with the usual norms are defined by

$$
\|u\|_{H^{r}(\Omega)}^{2}=\sum_{0 \leq|\alpha| \leq r}\left\|\partial^{\alpha} u\right\|_{L^{2}(\Omega)}^{2}, \quad \partial^{\alpha} u=\frac{\partial^{|\alpha|}}{\partial x_{1}^{\alpha_{1}} \partial x_{2}^{\alpha_{2}}} u
$$

where $\alpha=\left(\alpha_{1}, \alpha_{2}\right)$ is a non-negative integer pair, $|\alpha|=\alpha_{1}+\alpha_{2} ;\|\cdot\|_{L^{2}(\Omega)}$ denotes the usual norm in $L^{2}(\Omega)$ or $L^{2}(\Omega)^{2}$. The semi-norm $|\cdot|_{H^{r}(\Omega)}$ for $u \in H^{r}(\Omega)$ is defined via

$$
|u|_{H^{r}(\Omega)}^{2}=\sum_{|\alpha|=r}\left\|\partial^{\alpha} u\right\|_{L^{2}(\Omega)}^{2} .
$$

Let $H(\operatorname{div}, \Omega)$ denote the space of vector functions such that

$$
H(\operatorname{div}, \Omega):=\left\{\psi \in\left(L^{2}(\Omega)\right)^{2}: \operatorname{div} \psi \in L^{2}(\Omega)\right\},
$$

where 'div' means the divergence: for $\psi=\left(\psi_{1}, \psi_{2}\right)^{T}$, $\operatorname{div} \psi=\frac{\partial \psi_{1}}{\partial x_{1}}+\frac{\partial \psi_{2}}{\partial x_{2}}$.

In this paper, we consider the error estimate for an FEM applied to Poisson's equation:

$$
-\Delta u=f \text { in } \Omega,\left.\quad u\right|_{\partial \Omega}=0,
$$

where $f \in L^{2}(\Omega)$ is a given function. We fix a function space

$$
V=H_{0}^{1}(\Omega):=\left\{v \in H^{1}(\Omega): v=0 \text { on } \partial \Omega\right\} .
$$

Here, $v=0$ on $\partial \Omega$ is in the trace sense. The variational formulation of the problem (1) is given as:

$$
\text { Find } u \in V \text { satisfying }(\nabla u, \nabla v)=(f, v), \quad \forall v \in V .
$$

Let $\mathcal{T}^{h}$ be a proper triangulation of $\Omega$. The Lagrange finite element space $V_{h, k}(\subset V)$ will be used to find the approximate solution of (1). The function in $V_{h, k}$ is continuous over the domain and its restriction to each element is a polynomial of degree $\leq k$. The Ritz-Galerkin method solves the variational problem (2) in $V_{h, k}$,

Find $u_{h} \in V_{h, k}$ satisfying $\left(\nabla u_{h}, \nabla v_{h}\right)=\left(f, v_{h}\right), \quad \forall v_{h} \in V_{h, k}$. 
Riesz's representation theorem assures the existence and uniqueness of solutions $u \in V$ and $u_{h} \in V_{h, k}$.

Let $\mathcal{P}_{h}$ be the orthogonal projection operator from $V$ onto $V_{h, k}$ with respect to the inner product $(\nabla \cdot, \nabla \cdot)$. Then, setting $u_{h}:=\mathcal{P}_{h} u$, we have

$$
\left(\nabla\left(u-u_{h}\right), \nabla v_{h}\right)=0, \quad \forall v_{h} \in V_{h, k} .
$$

If the solution $u$ of (1) is regular enough, classical error estimation theory through interpolation function error estimation implies

$$
\left|u-u_{h}\right|_{H^{m}} \leq C h^{k+1-m}|u|_{H^{k+1}}, \text { for } m=0,1 ; k \geq 1 .
$$

For a solution with lower regularity, such as $u \in H^{\sigma}(\Omega)(\sigma \in[1,2))$, the convergence order is lower; see, e.g., Chapter 8.3 of [4], from which we have

$$
\left|u-u_{h}\right|_{H^{1}} \leq C h^{\sigma-1}|u|_{H^{\sigma}}, \quad\left\|u-u_{h}\right\|_{L^{2}} \leq C h^{2(\sigma-1)}|u|_{H^{\sigma}},
$$

One can introduce singular functions as trial functions to recover the convergence order; see, e.g., [4]. In particular, for a polygonal domain with re-entrant right corners, e.g., the L-shaped domain, K. Kobayashi gives an explicit a priori estimate along with the use of singular functions [5].

In this paper, we consider an a prior error estimate for the Poisson problem with a solution of lower regularity, without using singular functions, as follows:

$$
\left|u-u_{h}\right|_{H^{1}} \leq M_{h}\|f\|_{L^{2}}, \quad\left\|u-u_{h}\right\|_{L^{2}} \leq M_{h}^{2}\|f\|_{L^{2}},
$$

where the quantity $M_{h}$, having an explicit value, is independent of $f$. The dependency of $M_{h}$ on $k$, $h$ and the solution regularity is examined in the numerical computations found in Section 4.

\section{FEM error analysis for Poisson's equation}

Denote by $\tau: L^{2}(\Omega) \rightarrow H_{0}^{1}(\Omega)$ the linear operator that maps $f$ to the solution $u$ of (2). The optimal value of the quantity $M_{h}$ in (3) is characterized by

$$
M_{h}=\sup _{f \in L^{2}(\Omega), f \neq 0} \frac{\left|\left(I-\mathcal{P}_{h}\right) \tau f\right|_{H^{1}}}{\|f\|_{L^{2}}} .
$$

In what follows, we provide an algorithm that gives the concrete value of $M_{h}$ for a higher degree finite element scheme.

\subsection{The finite element spaces}

We begin by introducing the Raviart-Thomas finite element space [6,7]. For each triangular element $K$, define by $P_{k}(K)$ the space of polynomials of degree less than or equal to $k$ on $K$. The RaviartThomas finite element space $R T_{k}$ is given by

$$
\begin{array}{r}
R T_{k}:=\left\{p_{h} \in\left(L^{2}(\Omega)\right)^{2}:\right. \\
\left.p_{h}\right|_{K}=\left(\begin{array}{c}
a_{k} \\
b_{k}
\end{array}\right)+c_{k} \cdot\left(\begin{array}{c}
x \\
y
\end{array}\right), a_{k}, b_{k}, c_{k} \in P_{k}(K), \\
\left.p_{h} \cdot n \text { is continuous on the inter-element boundaries. }\right\}
\end{array}
$$

The Raviart-Thomas finite element space is a finite dimensional subspace of $H(\operatorname{div}, \Omega)$. Furthermore, we define the space of piecewise polynomial functions on $K$ whose degree is less than or equal to $k$ :

$$
X_{h, k}:=\left\{g \in L^{2}(\Omega):\left.g\right|_{K} \in P_{k}(K)\right\} .
$$

Classical analysis shows that $\operatorname{div}\left(R T_{k}\right)=X_{h, k}$ (cf. Chapter IV.1 of [7]). For each $f_{h} \in X_{h, k}$, we define a subset of $R T_{k}$ by

$$
W_{f_{h}}:=\left\{p_{h} \in R T_{k}: \operatorname{div} p_{h}+f_{h}=0 \text { on each } K \in \mathcal{T}^{h}\right\}
$$


Define the orthogonal projection $\Pi_{h, k}: L^{2}(\Omega) \rightarrow X_{h, k}$ by

$$
\left(u-\Pi_{h, k} u, g_{h}\right)=0, \quad \forall g_{h} \in X_{h, k} .
$$

The property of the orthogonality implies that

$$
\|u\|_{L^{2}}^{2}=\left\|\Pi_{h, k} u\right\|_{L^{2}}^{2}+\left\|u-\Pi_{h, k} u\right\|_{L^{2}}^{2}, \quad \forall u \in L^{2}(\Omega) .
$$

To give an error estimate for $\left\|u-\Pi_{h, k} u\right\|_{L^{2}}$, let us consider the restriction of $\Pi_{h, k}$ to the triangular element $K$ of $\mathcal{T}^{h}$. Let $\Pi_{K, k}$ be the orthogonal $L^{2}$ projection that projects $u \in L^{2}(K)$ onto $P_{k}(K)$. Define the constant $C_{k}(K)$ over a triangular element $K$ as follows:

$$
C_{k}(K):=\sup _{u \in H^{1}(K),|u|_{H^{1}(K)} \neq 0} \frac{\left\|u-\Pi_{K, k} u\right\|_{L^{2}}}{|u|_{H^{1}}}
$$

Define $C_{h, k}$ by $C_{h, k}:=\max _{K \in \mathcal{T}^{h}} C_{k}(K)$. Then, the error estimate for $\left(u-\Pi_{h, k} u\right)$ is given by

$$
\left\|u-\Pi_{h, k} u\right\|_{L^{2}} \leq C_{h, k}|u|_{H^{1}} \text { if } u \in H^{1}(\Omega) .
$$

From the definition of the projection $\Pi_{h, k}$, we know $\left\|u-\Pi_{h, k+1} u\right\|_{L^{2}(K)} \leq\left\|u-\Pi_{h, k} u\right\|_{L^{2}(K)}$ for $k \geq 0$; thus, $C_{k+1}(K) \leq C_{k}(K)$. In the case $k=0$, the inverse of $C_{0}(K)$ is just the square root of the minimal positive eigenvalue of the Laplacian over $K$ with Neumann boundary condition $[8,9]$.

\subsection{A computable a priori error estimate}

We now turn to the main result of the paper: the calculation of the quantity $M_{h}$. We will apply the framework of [1] to high degree finite elements to give a sharp estimate for $M_{h}$. For the sake of simplicity, we denote $R T_{k-1}$ by $W_{h}, X_{h, k-1}$ by $X_{h}$, and $V_{h, k}$ by $V_{h}$ for FEM spaces sharing the same degree $k, k \geq 1$.

We extend the definition of $\kappa_{h}$ in [1] to one over general finite element spaces:

$$
\kappa_{h}:=\max _{0 \neq f_{h} \in X_{h}} \min _{\left\{v_{h}, p_{h}\right\} \in V_{h} \times W_{f_{h}}} \frac{\left\|p_{h}-\nabla v_{h}\right\|_{L^{2}}}{\left\|f_{h}\right\|_{L^{2}}} .
$$

We denote by $\kappa_{h, k}$ the quantity $\kappa_{h}$ in the case of FEM spaces of degree $k$. This quantity was first introduced by X. Liu et al in [1], where the piecewise linear conforming finite element space and lowest order Raviart-Thomas mixed finite element are considered.

The quantity $\kappa_{h}$ is used to construct a computable a priori error estimate. The following proofs in Lemma 1 and Theorem 1 are analogous to proofs given in [1]; here, however, more general finite element spaces are considered.

Lemma 1. Given $f_{h} \in X_{h}$, let $\bar{u} \in H_{0}^{1}(\Omega)$ and $\bar{u}_{h} \in V_{h}$ be the solutions of problems,

$$
(\nabla \bar{u}, \nabla v)=\left(f_{h}, v\right), \quad \forall v \in V \quad \text { and }\left(\nabla \bar{u}_{h}, \nabla v_{h}\right)=\left(f_{h}, v_{h}\right), \quad \forall v_{h} \in V_{h},
$$

respectively. Then we have the following error estimate in terms of the quantity $\kappa_{h}$ :

$$
\left|\bar{u}-\bar{u}_{h}\right|_{H^{1}} \leq \kappa_{h}\left\|f_{h}\right\|_{L^{2}} .
$$

Proof. From Prager-Synge's theorem [10], for any $v_{h} \in V_{h}$ and any $p_{h} \in W_{f_{h}}$, we have

$$
\left\|\nabla \bar{u}-\nabla v_{h}\right\|_{L^{2}}^{2}+\left\|\nabla \bar{u}-p_{h}\right\|_{L^{2}}^{2}=\left\|p_{h}-\nabla v_{h}\right\|_{L^{2}}^{2} .
$$

This is called the hypercircle equation, and it implies the inequality

$$
\left\|\nabla \bar{u}-\nabla v_{h}\right\|_{L^{2}} \leq\left\|p_{h}-\nabla v_{h}\right\|_{L^{2}}, \quad \forall v_{h} \in V_{h}, \forall p_{h} \in W_{f_{h}} .
$$

From the minimization principle, we obtain the error estimate, 


$$
\left\|\nabla \bar{u}-\nabla \bar{u}_{h}\right\|_{L^{2}} \leq \min _{v_{h} \in V_{h}}\left\|\nabla \bar{u}-\nabla v_{h}\right\|_{L^{2}} \leq \min _{\left\{v_{h}, p_{h}\right\} \in V_{h} \times W_{f_{h}}}\left\|p_{h}-\nabla v_{h}\right\|_{L^{2}} .
$$

By the definition of $\kappa_{h}$, we then have

$$
\left\|\nabla\left(\bar{u}-\bar{u}_{h}\right)\right\|_{L^{2}} \leq \kappa_{h}\left\|f_{h}\right\|_{L^{2}} .
$$

Theorem 1. Given $f \in L^{2}(\Omega)$, let $u \in V$ be the solution of

$$
(\nabla u, \nabla v)=(f, v), \quad \forall v \in V
$$

and $\mathcal{P}_{h} u$ is the projection of $u$ onto $V_{h}$. Let

$$
M_{h}:=\sqrt{\left(C_{h, k-1}\right)^{2}+\kappa_{h}^{2}} .
$$

We have the following error estimate:

$$
\left|u-\mathcal{P}_{h} u\right|_{H^{1}} \leq M_{h}\|f\|_{L^{2}}, \quad\left\|u-\mathcal{P}_{h} u\right\|_{L^{2}} \leq M_{h}\left|u-\mathcal{P}_{h} u\right|_{H^{1}} \leq M_{h}{ }^{2}\|f\|_{L^{2}} .
$$

Proof. Let $\bar{u}$ and $\bar{u}_{h}$ be as defined in Lemma 1, where $f_{h}$ is chosen to be $f_{h}:=\Pi_{h, k-1} f \in X_{h}$. The minimization principle yields $\left|u-\mathcal{P}_{h} u\right|_{H^{1}} \leq\left|u-\bar{u}_{h}\right|_{H^{1}}$. Re-writing $u-\bar{u}_{h}$ as $(u-\bar{u})+\left(\bar{u}-\bar{u}_{h}\right)$, we find that

$$
\left|u-\mathcal{P}_{h} u\right|_{H^{1}} \leq\left|u-\bar{u}_{h}\right|_{H^{1}} \leq|u-\bar{u}|_{H^{1}}+\left|\bar{u}-\bar{u}_{h}\right|_{H^{1}} .
$$

First, we consider the estimate for $|u-\bar{u}|_{H^{1}}$. From the definitions of $u$ and $\bar{u}$, it follows that, for $\forall v \in V$,

$$
(\nabla(u-\bar{u}), \nabla v)=\left(f-\Pi_{h, k-1} f, v\right)=\left(\left(I-\Pi_{h, k-1}\right) f,\left(I-\Pi_{h, k-1}\right) v\right) .
$$

Putting $v:=u-\bar{u}$ and applying the error estimate for the projection $\Pi_{h, k-1}$ in (5), we have

$$
\begin{aligned}
|u-\bar{u}|_{H^{1}}^{2} & \leq\left\|\left(I-\Pi_{h, k-1}\right) f\right\|_{L^{2}}\left\|\left(I-\Pi_{h, k-1}\right)(u-\bar{u})\right\|_{L^{2}} \\
& \leq\left\|\left(I-\Pi_{h, k-1}\right) f\right\|_{L^{2}} \cdot C_{h, k-1}|u-\bar{u}|_{H^{1}} .
\end{aligned}
$$

Hence, we obtain

$$
|u-\bar{u}|_{H^{1}} \leq C_{h, k-1}\left\|\left(I-\Pi_{h, k-1}\right) f\right\|_{L^{2}} .
$$

From (4), (7) and (8), $\left|u-\mathcal{P}_{h} u\right|_{H^{1}}$ satisfies

$$
\begin{aligned}
\left|u-\mathcal{P}_{h} u\right|_{H^{1}} & \leq|u-\bar{u}|_{H^{1}}+\left|\bar{u}-\bar{u}_{h}\right|_{H^{1}} \\
& \leq \kappa_{h}\left\|\Pi_{h, k-1} f\right\|_{L^{2}}+C_{h, k-1}\left\|\left(I-\Pi_{h, k-1}\right) f\right\|_{L^{2}} \\
& \leq \sqrt{\left(C_{h, k-1}\right)^{2}+\kappa_{h}^{2}}\|f\|_{L^{2}} .
\end{aligned}
$$

Finally, the estimate for $\left\|u-\mathcal{P}_{h} u\right\|_{L^{2}}$ easily follows from Aubin-Nitsche's method.

\subsection{Computation of $\kappa_{h}$}

Next, we turn to evaluating the quantity $\kappa_{h}$ defined in (6). The computation of $\kappa_{h}$ is accomplished in two steps. First, for a fixed $f_{h} \in X_{h}$, letting $\bar{u}$ be the solution corresponding to $f_{h}$, we find the functions $\bar{u}_{h}$ and $p_{h}$ that solve the optimization problem:

$$
\min _{\left\{\bar{u}_{h}, p_{h}\right\} \in V_{h} \times W_{f_{h}}}\left\|p_{h}-\nabla \bar{u}_{h}\right\|_{L^{2}}^{2}=\min _{\left\{\bar{u}_{h}, p_{h}\right\} \in V_{h} \times W_{f_{h}}}\left\|\nabla \bar{u}-p_{h}\right\|_{L^{2}}^{2}+\left\|\nabla \bar{u}-\nabla \bar{u}_{h}\right\|_{L^{2}}^{2} .
$$

Since $\bar{u}_{h}$ and $p_{h}$ can be selected independently, solving the above optimization problem is equivalent to solving the following two optimization problems:

$$
\min _{p_{h} \in W_{f_{h}}}\left\|\nabla \bar{u}-p_{h}\right\|_{L^{2}}^{2}, \quad \min _{\bar{u}_{h} \in V_{h}}\left\|\nabla \bar{u}-\nabla \bar{u}_{h}\right\|_{L^{2}}^{2} .
$$

Classical FEM error analysis implies that the minimizers are given by the solutions of the following two problems: 
a) Find $p_{h} \in W_{h}$ and $\rho_{h} \in X_{h}$ such that

$$
\begin{cases}\left(p_{h}, q_{h}\right)+\left(\rho_{h}, \operatorname{div} q_{h}\right)=0, & \forall q_{h} \in W_{h} \\ \left(\operatorname{div} p_{h}, g_{h}\right)+\left(f_{h}, g_{h}\right)=0, & \forall g_{h} \in X_{h} .\end{cases}
$$

b) Find $\bar{u}_{h} \in V_{h}$ such that

$$
\left(\nabla \bar{u}_{h}, \nabla v_{h}\right)=\left(f_{h}, v_{h}\right), \quad \forall v_{h} \in V_{h} .
$$

Second, since the optimizers of (9) are uniquely determined for each $f_{h}$, the maximum of $\| p_{h}-$ $\nabla \bar{u}_{h}\left\|_{L^{2}} /\right\| f_{h} \|_{L^{2}}$ can be obtained by solving an eigenvalue problem in a finite dimensional space. Observe that the optimizer $\left\{p_{h}, u_{h}\right\}$ for (9) satisfies

$$
\left(\nabla \bar{u}_{h}, \nabla \bar{u}_{h}\right)=\left(f_{h}, \bar{u}_{h}\right), \quad\left(\nabla \bar{u}_{h}, p_{h}\right)=-\left(\bar{u}_{h}, \operatorname{div} p_{h}\right)=\left(\bar{u}_{h}, f_{h}\right),
$$

and

$$
\left(p_{h}, p_{h}\right)=-\left(\rho_{h}, \operatorname{div} p_{h}\right)=\left(\rho_{h}, f_{h}\right)
$$

Thus, we have

$$
\begin{aligned}
\left\|p_{h}-\nabla \bar{u}_{h}\right\|_{L^{2}}^{2} & =\left(p_{h}, p_{h}\right)-2\left(\nabla \bar{u}_{h}, p_{h}\right)+\left(\nabla \bar{u}_{h}, \nabla \bar{u}_{h}\right) \\
& =\left(f_{h}, \rho_{h}-\bar{u}_{h}\right) .
\end{aligned}
$$

Defining $\bar{u}_{h}, \rho_{h}$ via $\bar{u}_{h}=K f_{h}$ and $\rho_{h}=H f_{h}$, we then have

$$
\kappa_{h}^{2}=\max _{f_{h} \in X_{h}} \frac{\left(f_{h},(H-K) f_{h}\right)}{\left(f_{h}, f_{h}\right)} .
$$

We note that the matrix expression of the numerator of (10) is a full-matrix, which makes solving the matrix eigenvalue problem extremely computationally intensive if verified bound of $\kappa_{h}$ is required. However, for purpose of approximate computation of $\kappa_{h}$, the explicit form of matrices corresponding to $H$ and $K$ is not needed. Thus, both computer memory and computing time can be dramatically saved. For a detailed description of the computation of $\kappa_{h}$, we refer the reader to [1].

\section{Computational results of a priori error estimate}

In this section, we give illustrative numerical examples of computable a priori error estimates for Poisson's equation. The dependence of the quantity $M_{h}$ on the mesh size, FEM degree and the solution regularity is discussed within these numerical results.

\subsection{FEM of varying mesh sizes and degrees}

First, we investigate error estimates for FEM of different mesh sizes and degrees. The degree of FEM spaces varies from $k=1$ to $k=6$. The DOLFIN package of the FEniCS [11] project is used to implement these higher degree FEM spaces. As interval arithmetic is not supported by the DOLFIN package, the computational results obtained exhibit some rounding error.

We fix our domain as the L-shaped region $(-1,1) \times(-1,1) \backslash[0,1] \times[-1,0]$. Since explicit computations of $C_{h, k}(k=1,2,3)$ are not currently known, we focus on the evaluation of quantity $C_{h, 0}$. In fact, $C_{h, 0}$ has the optimal convergence order and converges to 0 faster than $\kappa_{h, k}$.

In Fig. 1, we display the values of $\kappa_{h, k}$ versus the square root of the degrees of freedom of the FEM spaces $V_{h, k}$. The data on the same solid lines is from the computation of the same FEM degree using different mesh sizes. It is evident that the value of $\kappa_{h, k}$ converges to zero rapidly if we vary both the mesh size and the FEM degree. However, for a fixed degree of FEM spaces, the convergence order of $\kappa_{h, k}$, denoted by $\gamma$, is substantially smaller than 1, even if higher degree FEMs are adopted; see Fig. 2. 


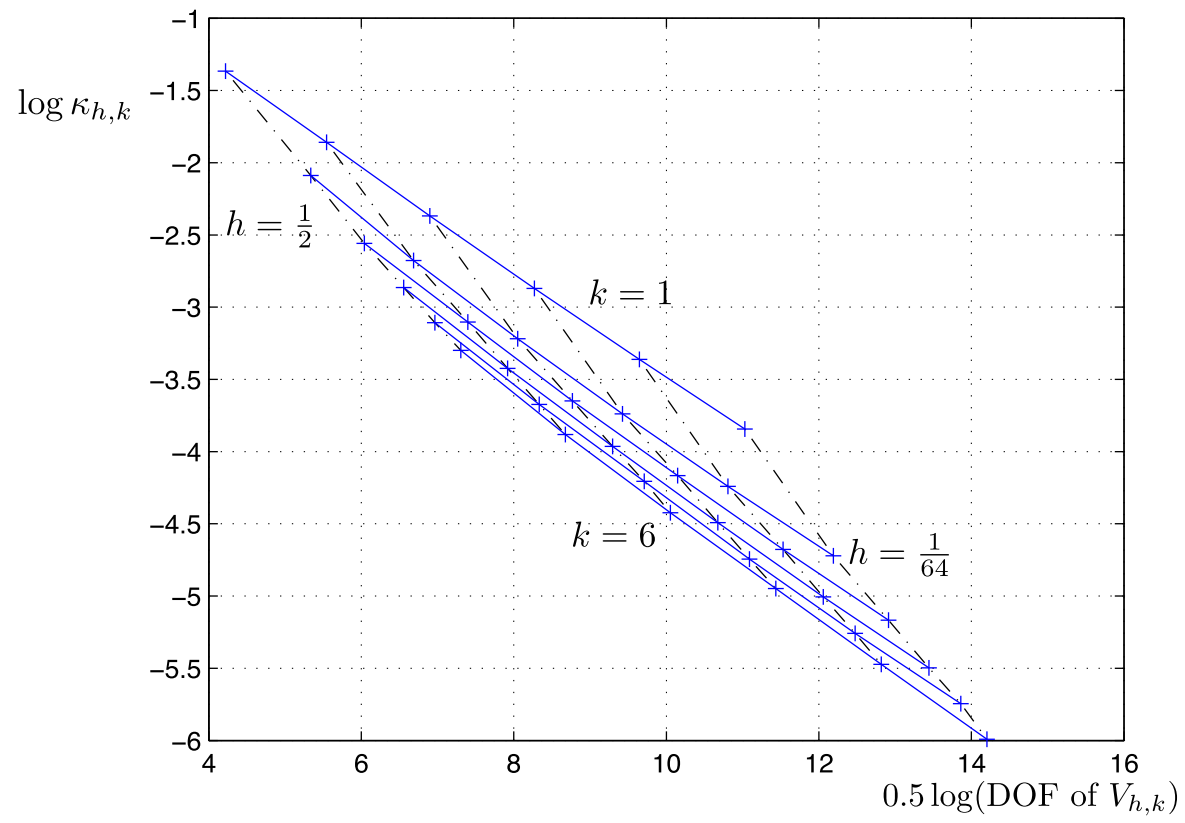

Fig. 1. Convergence of $\kappa_{h, k}$ v.s. degree of freedom(DOF) of $V_{h, k}$.

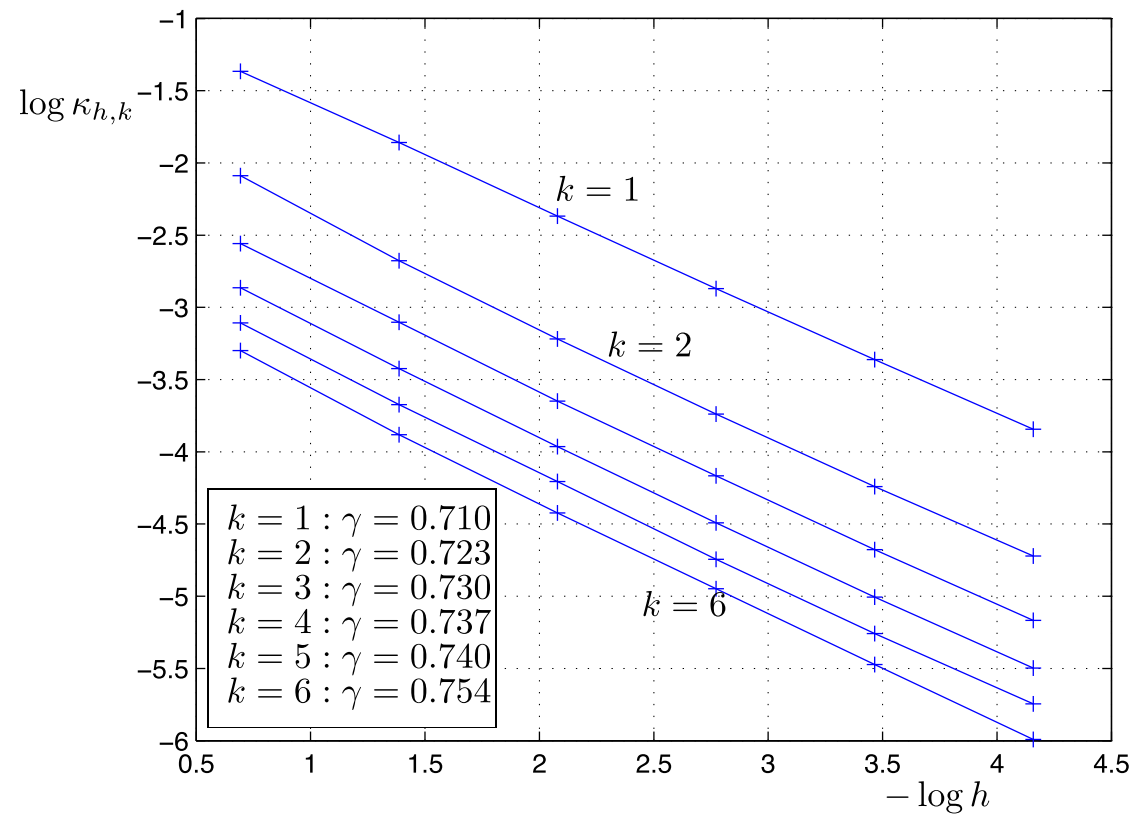

Fig. 2. Convergence of $\kappa_{h, k}$ versus mesh size $h$ and FEM degree $k$.

\subsection{Boundary value problems of varying singularity}

We define $\Omega_{m}(m=1,2,3,4)$, domains corresponding to re-entrant corners of different angles (see Fig. 3 and Fig. 4), as follows:

$$
\Omega_{m}=(-1,1)^{2} \cap\left\{(r \cos \theta, r \sin \theta): 0 \leq \theta \leq \frac{3+m}{4} \pi, 0<r<\sqrt{2}\right\} \quad(m=1,2,3,4) .
$$

Evidently, only the rectangle $\Omega_{1}$ is a convex domain. For Poisson's problem over the above domains, we implement the Raviart-Thomas element spaces $R T_{0}$ and $R T_{1}$ with interval computation. All computations in this subsection are carried out using MATLAB 2013a with a toolbox for verified numerical computations, INTLAB ver.7 [12].

First, we consider the a priori error estimate in the case of the domain $\Omega_{1}$. For the FEM spaces of degree $k=1$, it is well known that an explicit a priori estimate can be given by applying the 

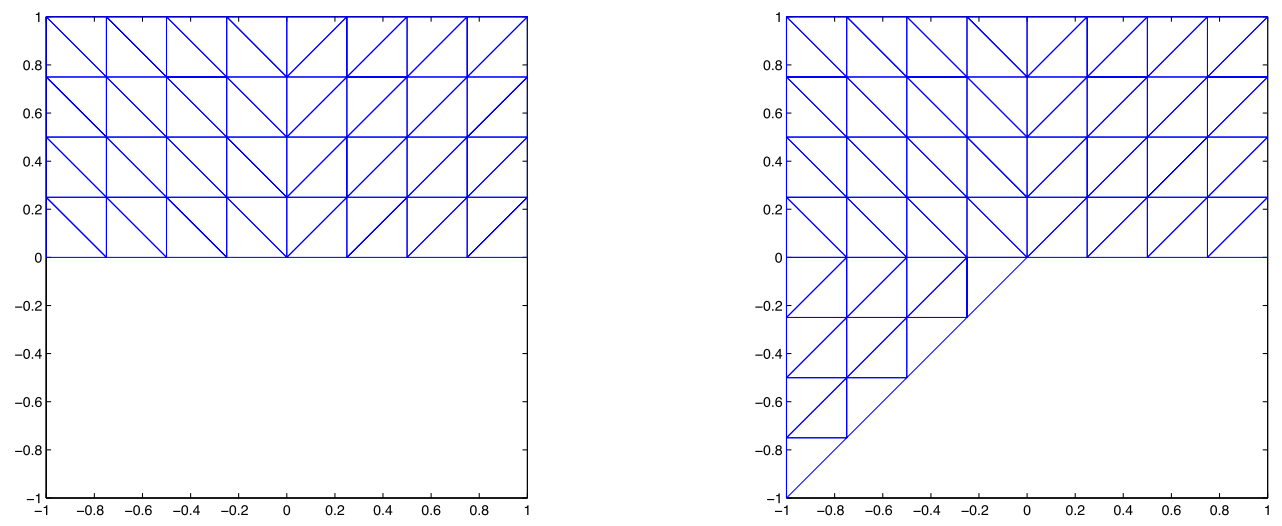

Fig. 3. Domains $\Omega_{1} \& \Omega_{2}$ with triangular meshes.
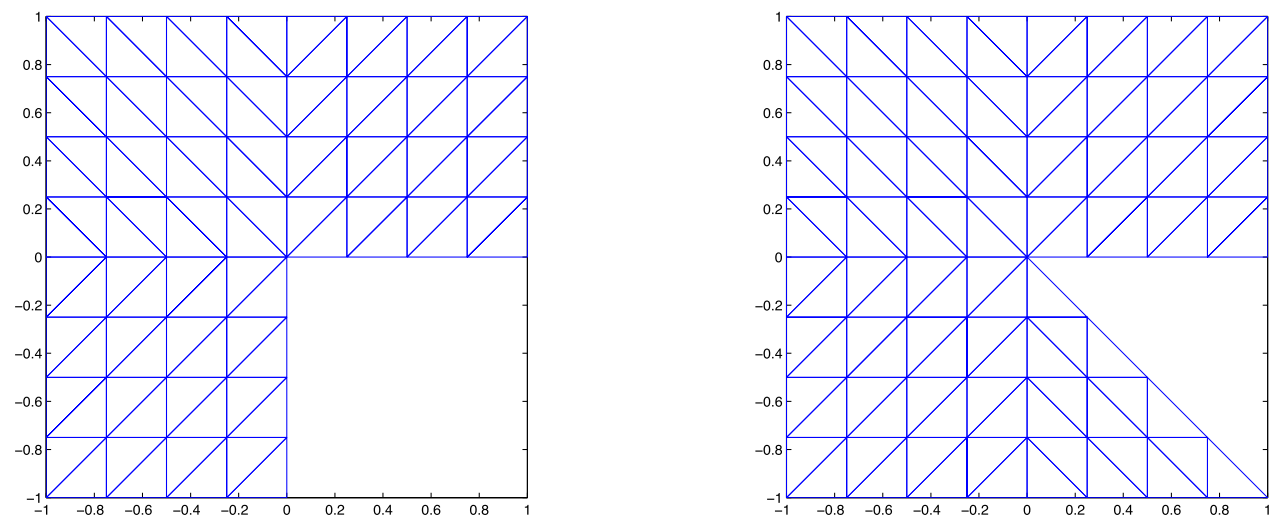

Fig. 4. Domains $\Omega_{3} \& \Omega_{4}$ with triangular meshes.

Table I. A priori estimation for $k=1,2$ on $\Omega_{1}$.

\begin{tabular}{c||cccc|cccc}
\hline$h$ & $M_{h, 1}$ & order & $M_{h, 1}(f)$ & order & $M_{h, 2}$ & order & $M_{h, 2}(f)$ & order \\
\hline $1 / 2$ & $2.61 \mathrm{E}-1$ & - & $1.81 \mathrm{E}-1$ & - & $1.90 \mathrm{E}-1$ & - & $4.19 \mathrm{E}-2$ & - \\
$1 / 4$ & $1.37 \mathrm{E}-1$ & 0.93 & $9.8 \mathrm{E}-2$ & 0.89 & $9.63 \mathrm{E}-2$ & 0.98 & $1.26 \mathrm{E}-2$ & 1.73 \\
$1 / 8$ & $7.08 \mathrm{E}-2$ & 0.95 & $5.0 \mathrm{E}-2$ & 0.96 & $4.84 \mathrm{E}-2$ & 0.99 & $3.60 \mathrm{E}-3$ & 1.81 \\
$1 / 16$ & $3.65 \mathrm{E}-3$ & 0.96 & $2.5 \mathrm{E}-2$ & 0.99 & $2.43 \mathrm{E}-2$ & 1.00 & $9.96 \mathrm{E}-4$ & 1.85 \\
$1 / 32$ & $1.85 \mathrm{E}-4$ & 0.98 & $1.3 \mathrm{E}-2$ & 1.00 & $1.21 \mathrm{E}-2$ & 1.00 & $2.72 \mathrm{E}-4$ & 1.88 \\
\hline
\end{tabular}

interpolation error estimate for the solution $u \in H^{2}(\Omega)$; the explicit interpolation error estimate can be found in F. Kikuchi and X. Liu [8] and K. Kobayashi [13]. We use the value of $\kappa_{h, k}$ to construct the a priori estimate in this subsection. Since the quantity $M_{h, k}$ is defined using the supremum over all $f \in L^{2}(\Omega)$, the convergence order of $M_{h, k}$ is at most one, even if a higher degree FEM is employed. Thus, the convergence order of $M_{h, k}$ is precisely one, a fact that is confirmed by the computed results in Table I and Figs. 5 and 6.

For a concrete $f$, we can define the a priori estimate as

$$
\left|u-u_{h}\right|_{H^{1}} \leq M_{h, k}(f)\|f\|_{L^{2}}
$$

where $M_{h, k}(f)=\sqrt{C_{h, k}^{2}+\kappa_{h, k}^{2}(f)} ; \kappa_{h, k}(f)$ is defined by,

$$
\kappa_{h, k}(f)=\min _{\left\{v_{h}, p_{h}\right\} \in V_{h, k} \times W_{f_{h}}} \frac{\left|\nabla v_{h}-p_{h}\right|_{L^{2}}}{\left\|f_{h}\right\|_{L^{2}}} \quad\left(f_{h}:=\Pi_{h, k-1} f\right) .
$$

Thus, $M_{h, k}(f)$ can be smaller in value than $M_{h, k}$, and a better convergence order is expected. The minimizer $\left\{v_{h}, p_{h}\right\}$ in the definition of $\kappa_{h, k}(f)$ is just given by the solutions of problem $\left.\mathbf{a}\right)$ and $\left.\mathbf{b}\right)$ in Section 3.3. In Table I, for $f \equiv 1$, we show the a priori estimates for FEM spaces of high degree; the convergence orders tend to 1.0 and 2.0 for $k=1,2$, respectively. 


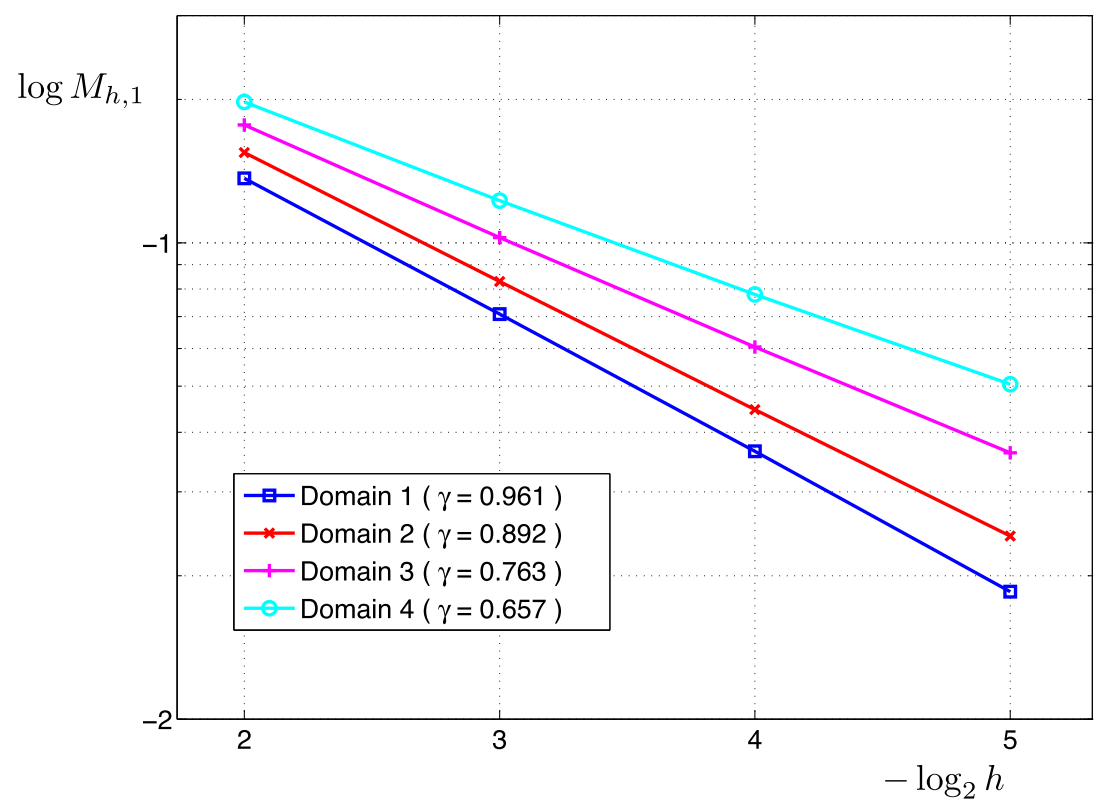

Fig. 5. $M_{h, 1}$ on $\Omega_{m},(m=1, \ldots, 4)$.

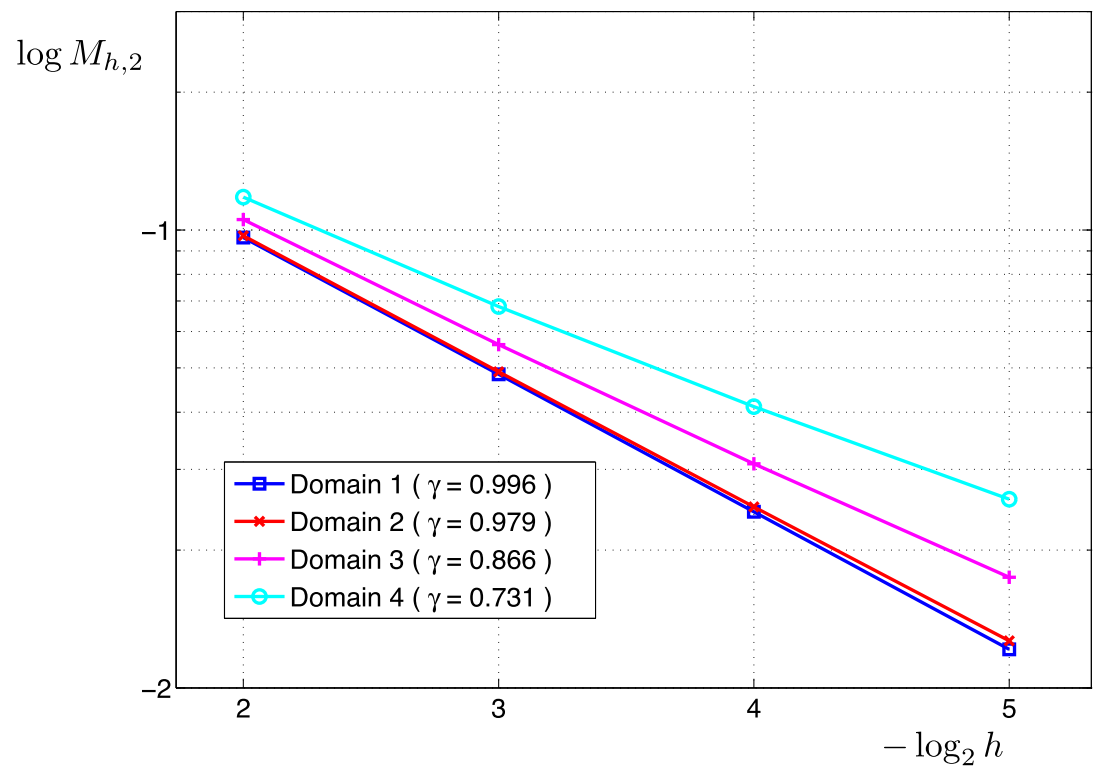

Fig. 6. $M_{h, 2}$ on $\Omega_{m},(m=1, \ldots, 4)$.

Next, we consider the cases of the domains $\Omega_{m}(m=1, \ldots, 4)$. In Figs. 5 and 6 , using double logarithmic plots, we display the values of $M_{h, k}$ for the FEM spaces of degree $k=1,2$ on $\Omega_{m}(m=$ $1, \ldots, 4)$ versus the mesh size $h$. It is well-known that the solutions over the domains $\Omega_{m},(m=2,3,4)$ may include parts with singularities:

$$
u=u_{s}+u_{0}
$$

where $u_{0}$ is a function in $H^{2}(\Omega)$ and $u_{s}$ may have a singularity as bad as

$$
u_{s}=r^{w_{i}} \sin w_{i} \theta
$$

with $w_{i}=4 / 5,2 / 3,4 / 7$ for $\Omega_{2}, \Omega_{3}$ and $\Omega_{4}$, respectively (cf. Chapter 8 of [4]). From the computational results in Fig. 5 and Fig. 6, it may be observed that a worse singularity leads to a lower convergence order, and a higher degree FEM method can help improve the convergence order. 


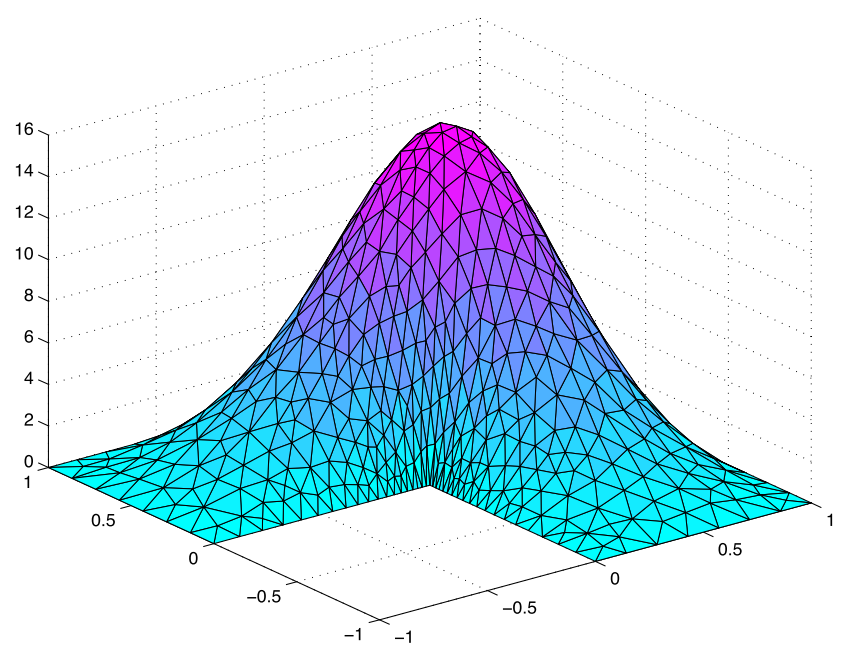

Fig. 7. A sketch of $\hat{u} \in V_{h, 2}$.

Table II. Verification computation for (11).

\begin{tabular}{ccccccc}
\hline$h_{\max }$ & $h_{\min }$ & $M_{h, 2}$ & $\beta$ & $\delta_{h}$ & $\beta^{2} \delta_{h} \eta$ & $\rho$ \\
\hline $9.088 \times 10^{-2}$ & $2.052 \times 10^{-3}$ & $2.589 \times 10^{-2}$ & 6.002 & $1.501 \times 10^{-1}$ & 0.613 & Failed \\
$4.333 \times 10^{-2}$ & $2.024 \times 10^{-3}$ & $1.246 \times 10^{-2}$ & 5.395 & $1.457 \times 10^{-1}$ & 0.481 & 1.309 \\
$8.539 \times 10^{-2}$ & $1.017 \times 10^{-3}$ & $2.452 \times 10^{-2}$ & 5.914 & $1.101 \times 10^{-1}$ & 0.437 & $9.594 \times 10^{-1}$ \\
\hline
\end{tabular}

\section{Applications to semilinear elliptic problems}

As an application of our computable a priori error estimates, we consider a semilinear elliptic problem. For such problems, a verified computation method has been developed to give a proof for the existence and local uniqueness of a solution based on Newton-Kantorovich's theorem; see, e.g., [14, 15] for the detailed procedure. The computable a priori error estimate plays an important role in implementing the verified computation.

In what follows, we study a semilinear elliptic problem. Let $\Omega=\left\{\left(x_{1}, x_{2}\right):(-1,1)^{2} \backslash[-1,0]^{2}\right\} \subset \mathbf{R}^{2}$ be an L-shaped domain with boundary $\partial \Omega$. We consider the following nonlinear equation:

$$
-\Delta u=u^{2} \text { in } \Omega,\left.\quad u\right|_{\partial \Omega}=0
$$

An approximate solution $\hat{u} \in V_{h, 2}$ is given by quadratic conforming finite elements on a non-uniform triangulation. The shape of $\hat{u}$ is displayed in Fig. 7. As the lack of $H^{2}$-regularity occurs at the re-entrant corner of the L-shaped domain, we refine the mesh around that corner.

In Table II, we present the quantities needed to construct the proof for (11). Here, $h_{\max }$ and $h_{\text {min }}$ denote, respectively, the maximum and minimum edge length of the triangulation. The quantity $M_{h, 2}$ is obtained by our proposed method introduced in Section 3. The sufficient condition in NewtonKantorovich's theorem to ensure the existence of a solution is: $\beta^{2} \delta_{h} \eta \leq 1 / 2$, where $\eta$ is the Lipschitz constant of the Fréchet derivative of the nonlinear term, $\beta$ is a norm estimate of the linearized inverse discussed in [15], and $\delta_{h}$ denotes a residual estimate described in [14]. If the condition $\beta^{2} \delta_{h} \eta \leq 1 / 2$ holds, then the existence and local uniqueness of an exact solution $u$ is assured in the ball centered at $\hat{u}$ with radius $\rho$, that is, $|u-\hat{u}|_{H^{1}} \leq \rho$. Our computations show that, around the approximate solution $\hat{u}$ shown in Fig. 7, there exists an exact solution of (11).

\section{Acknowledgments}

The authors express their sincere thanks to referees for their valuable comments and precise reviewing. The second author is partially supported by Grant-in-Aind for Young Scientists (B) (No.23740092) from Japan Society for the Promotion of Science. 


\section{References}

[1] X. Liu and S. Oishi, "Verified eigenvalue evaluation for elliptic operator on arbitrary polygonal domain," SIAM J. Numer. Anal., vol. 51, no. 3, pp. 1634-1654, 2013.

[2] N. Yamamoto and M. Nakao, "Numerical verifications of solutions for elliptic equations in nonconvex polygonal domains," Numer. Math., vol. 65, no. 1, pp. 503-521, 1993.

[3] I. Babuska and S. Manil, "The p and h-p versions of the finite element method, basic principles and properties," SIAM review, vol. 36, no. 4, pp. 578-632, 1994.

[4] G. Strang and G. Fix., An Analysis of the Finite Element Method, 2ed, Wellesley-Cambridge Press, 2008.

[5] K. Kobayashi, "A constructive A priori error estimation for finite element discretizations in a non-convex domain using singular functions," Japan J. Indust. Appl. Math., vol. 26, pp. 493-516, 2009.

[6] P. Raviart and J. Thomas, "A mixed finite element method for 2-nd order elliptic problems," in Mathematical Aspects of Finite Element Methods, vol. 606 of Lecture Notes in Mathematics, eds. Ilio Galligani and Enrico Magenes, pp. 292-315, Springer Berlin/Heidelberg, 1977.

[7] F. Brezzi and M. Fortin, Mixed and hybrid finite element methods, vol. 15 of Springer Series in Computational Mathematics, Springer-Verlag, New York, 1991.

[8] F. Kikuchi and X. Liu, "Estimation of interpolation error constants for the $P_{0}$ and $P_{1}$ triangular finite elements," Comput. Methods Appl. Mech. Engrg., vol. 196(37-40), pp. 3750-3758, 2007.

[9] X. Liu and F. Kikuchi, "Analysis and estimation of error constants for $P_{0}$ and $P_{1}$ interpolations over triangular finite elements," J. Math. Sci. Univ. Tokyo, vol. 17, pp. 27-78, 2010.

[10] W. Prager and J.L. Synge, "Approximations in elasticity based on the concept of function space," Quart. Appl. Math., vol. 5, pp. 241-269, 1947.

[11] A. Logg and G.N. Wells, "DOLFIN: Automated Finite Element Computing," ACM Trans. on Math. Soft., vol. 37, no. 2, 2010.

[12] S.M. Rump, "INTLAB - INTerval LABoratory," in Developments in Reliable Computing, ed. Tibor Csendes, pp.77-104. Kluwer Academic Publishers, Dordrecht, 1999. http://www.ti3. tu-harburg.de/rump/.

[13] K. Kobayashi, "On the interpolation constants over triangular elements (in Japanese)," RIMS Kokyuroku, vol. 1733, pp. 58-77, 2011.

[14] A. Takayasu, X. Liu, and S. Oishi, "Verified computations to semilinear elliptic boundary value problems on arbitrary polygonal domains," NOLTA, vol. 4, no. 1, pp. 34-61, 2013.

[15] K. Tanaka, A. Takayasu, X. Liu, and S. Oishi, "Verified norm estimation for the inverse of linear elliptic operators using eigenvalue evaluation," to appear. 